\title{
Redox-addressable single-molecule junctions incorporating a persistent organic radical.
}

Saman Naghibi, a,\# Sara Sangtarash,,,\# Varshini J. Kumar,c,\# Jian-Zhong Wu, ${ }^{d}$ Martyna M. Judd, ${ }^{\mathrm{e}}$ Xiaohang Qiao, ${ }^{\mathrm{a}}$ Elena Gorenskaia, ${ }^{\mathrm{c}}$ Simon J. Higgins, ${ }^{\mathrm{a}}$ Nicholas Cox, ${ }^{\mathrm{e}}$ Richard J. Nichols, ${ }^{a,{ }^{*}}$ Hatef Sadeghi, ${ }^{\mathrm{b},{ }^{\star}}$ Paul J. Low, ${ }^{\mathrm{c},{ }^{\star}}$ and Andrea Vezzolia, ${ }^{\mathrm{a},{ }^{\star}}$

a) Department of Chemistry, University of Liverpool, Crown Street, Liverpool L69 7ZD, United Kingdom

b) School of Engineering, University of Warwick, Coventry CV4 7AL, United Kingdom

c) School of Molecular Sciences, University of Western Australia, Crawley, Western Australia 6009, Australia

d) School of Chemistry, South China Normal University, Guangzhou 510006, People's Republic of China

e) Research School of Chemistry, Australian National University, Canberra, ATC 2601, Australia

f) Stephenson Institute for Renewable Energy, University of Liverpool, Peach Street, Liverpool L69 7ZF, United Kingdom

\#: these authors contributed equally to this work.

*: corresponding authors: andrea.vezzoli@liverpool.ac.uk

paul.low@uwa.edu.au

hatef.sadeghi@warwick.ac.uk

nichols@liverpool.ac.uk 


\section{Abstract}

The integration of radical (open-shell) species into single-molecule junctions at non-cryogenic temperatures is a key to unlocking the potential of molecular electronics in further applications. While many efforts have been devoted to this issue, in the absence of a chemical or electrochemical potential the open-shell character is lost when in contact with the metallic electrodes. Here, the organic 6-oxo-verdazyl radical, which is stable at ambient temperatures and atmosphere, has been functionalised by aurophilic 4-thioanisole groups at the 1,5-positions and fabricated into a molecular junction using the scanning tunnelling microscope break-junction technique. The verdazyl moiety retains open-shell character within the junction even at room temperature, and electrochemical gating permits in-situ reduction of the verdazyl to the closed-shell anionic state in a single-molecule transistor configuration. In addition, the bias-dependent alignment of the open-shell resonances with respect to the electrode Fermi levels gives rise to purely electronically-driven rectifying behaviour. The demonstration of a verdazyl-based molecular junction capable of integrating radical character, transistor-like switching behaviour, and rectification in a single molecular component under ambient conditions paves the way for further studies of the electronic, magnetic, and thermoelectric properties of open-shell species. 


\section{Main Text}

Molecular radicals feature open-shell electronic structures (i.e. an odd number of electrons) and, as a consequence, organic radicals are often highly reactive and sensitive to atmospheric oxygen and moisture. Nevertheless, since Gomberg's successful synthesis of the triphenylmethyl (trityl) radical in $1900,{ }^{1}$ several organic open-shell compounds that are stable under ambient conditions have been prepared and characterised, and they have shown outstanding performances in electronic devices such as batteries, transistors and light-emitting diodes. ${ }^{2}$ When incorporated into metal | molecule | metal molecular junctions, the presence of open-shell orbitals results in sharp transport resonances in the transmission function $T(E)$ (the probability for an electron with given energy $E$ to tunnel through the molecular wire) near to the electrode Fermi level. ${ }^{3}$ These resonances are highly sought-after features in molecular electronics, ${ }^{4-6}$ imparting desirable properties such as rectification or non-linear behaviour. ${ }^{7}$ As the conductance $G$ of a molecular junction can be estimated using the Landauer formula $G=G_{0} T\left(E_{F}\right)$ where $G_{0}=2 e^{2} / h \cong 77.48 \mu S$, a high transmission coefficient (i.e. a resonance) at the Fermi level results in high charge transport efficiency. In addition, the high slope of $T(E)$ vs $E$ near the Fermi energy offered by radical systems is predicted to give rise to high Seebeck coefficients, leading to enticing prospects for the design of molecular thermoelectric materials, $6,8,9$ whilst the presence of a unpaired electron provides the basis from which to extend electronics to spintronics. ${ }^{10,11}$

Despite the rich promise of radical-based molecular electronics, attempts to date to incorporate persistent organic radicals into single-molecule junctions at room temperature have resulted in loss of the open-shell character, ${ }^{12}$ or in the predicted increase in charge transport efficiency failing to materialise ${ }^{13}$ and only experiments at cryogenic temperatures ${ }^{14,15}$ have been successful. These phenomena are not entirely surprising as the molecular components used had either a highly delocalised radical state (e.g. polyhalogenated trityl compounds) $)^{16,17}$ or a low oxidation potential (e.g. Blatter radical, $-0.29 \mathrm{~V}$ vs $\left.\mathrm{Fc} / \mathrm{Fc}^{+}\right),{ }^{12}$ which leads to electron transfer from the SOMO when in contact with the metallic electrodes, and hence quenching of the open-shell state. To overcome these issues, methods involving the in-situ generation of radical states has been successfully adopted, with electrochemical gating or chemical redox processes providing the required long-term stability of the molecular radical within the junction. ${ }^{18-22}$ The search for a single-molecule electronic device, based on 
a native, persistent radical species that is stable at room temperature and under ambient atmospheric conditions, is therefore ongoing.

In this letter, we demonstrate that the 6-oxoverdazyl derivative 1 maintains its radical character when fabricated into single-molecule junctions between two $\mathrm{Au}$ electrodes, showing a significantly enhanced conductance when compared with its closed-shell tetrazin-3-one precursor 2 (Figure 1). We attribute our finding to the localisation of the radical state on the nitrogen atoms of the oxoverdazyl core (Figure 1b), ${ }^{23}$ that allows 1 to maintain its open-shell nature even when coordinated to the two metallic nanoelectrodes at room temperature. The presence of a radical state in the single-molecule junction is unequivocally confirmed by reversible in situ electrochemistry of $\mathbf{1}$ within the junction, which can be exploited at the same time to engineer single-molecule transistor behaviour. Furthermore, technologically relevant properties such as rectification and nonlinear current-voltage behaviour are imparted to the single-molecule device by the presence of a strongly localised SOMO.

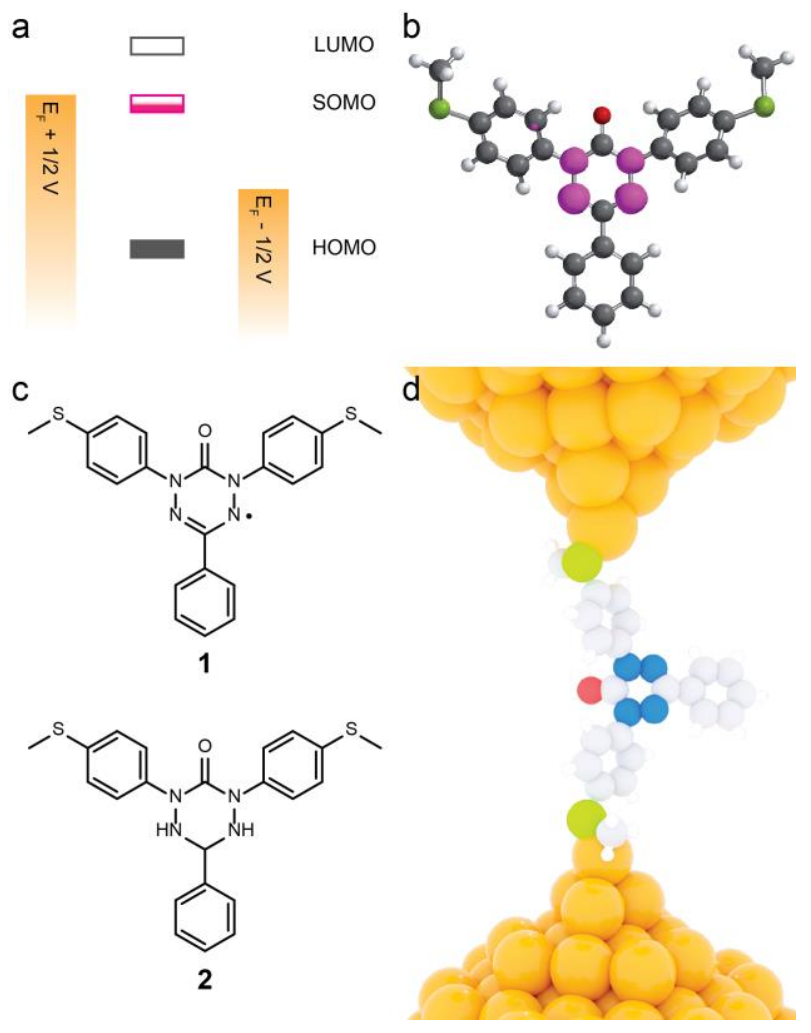

Figure 1: Rationale and proposed experiments. (a) Illustrative example of the energy level diagram for an open-shell material within a molecular junction. The SOMO (magenta) will sit in the HOMO-

LUMO gap and better aligned with the Fermi levels of the two metallic electrodes, resulting in higher conductance; (b) Spin density map (magenta) for the oxoverdazyl radical 1, with isosurfaces at 0.005 electrons per cubic Bohr (B3LYP/6-31G DFT, Spartan '18); (c) Structures of the compounds used in this study; and (d) cartoon depiction of 1 coordinated to two Au nanoelectrodes as a single-molecule junction. Atom colours in (b,d): $\mathrm{C}=$ grey; $\mathrm{H}=$ white; $\mathrm{O}=$ red; $\mathrm{S}=$ green; $\mathrm{N}=$ blue; $\mathrm{Au}=$ orange. 
The synthesis and characterisation of $\mathbf{1}$ and $\mathbf{2}$ are described elsewhere. ${ }^{24}$ Evidence of the persistent radical state of 1 is given by the presence of adsorption bands at $17300 \mathrm{~cm}^{-1}(\sim 580 \mathrm{~nm}$, corresponding to an optical bandgap of $2.13 \mathrm{eV})$ and $27600 \mathrm{~cm}^{-1}(\sim 360 \mathrm{~nm}, 3.44 \mathrm{eV})$, assigned to transitions involving singly-occupied orbitals, ${ }^{24-26}$ while 2 only shows an absorption band in the ultraviolet corresponding to a $\pi-\pi^{*}$ (HOMO-LUMO) transition (Figure 2a). In addition, continuouswave electron paramagnetic resonance (cw-EPR) spectroscopy of $\mathbf{1}$ exhibited a line-shape characteristic of 6-oxoverdazyl derivatives (Figure $2 b$ ), ${ }^{23,27-29}$, with a hyperfine structure that can be rationalized in terms of the unpaired electron spin coupling to two sets of equivalent $I=1$ nuclei (two sets of equivalent ${ }^{14} \mathrm{~N}$ atoms), consistent with the calculated distribution of the spin density over these atoms. EPR measurements of $\mathbf{1}$ adsorbed on a Au substrate confirm the retention of its open-shell character (Figure 2b, inset). Its spectrum has a simpler structure due to line broadening (from the loss of molecular tumbling) and increased delocalization of the unpaired spin (for further details see the SI).
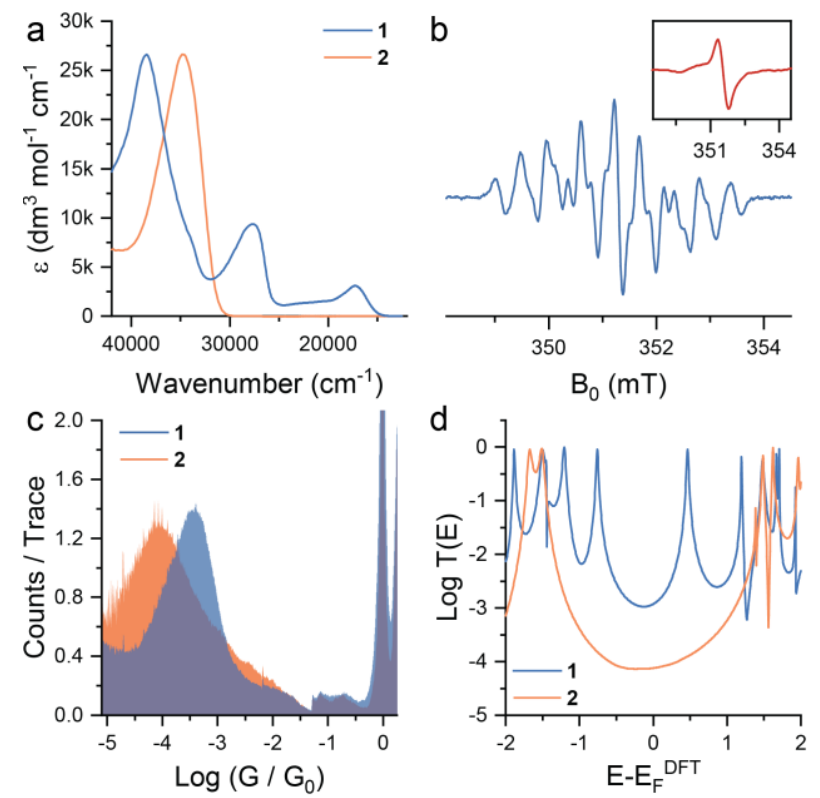

Figure 2: Experimental determination of open-shell properties of 1. (a) UV-Vis spectra of 1 and 2 in

$\mathrm{CH}_{2} \mathrm{Cl}_{2}$ solution; (b) cw-EPR (X-band $\sim 9.4 \mathrm{GHz}$ ) of 1 in solution $\left(1 \mathrm{mM}\right.$ in $\mathrm{CH}_{2} \mathrm{Cl}_{2}$, blue) and adsorbed on a Au substrate (inset, red); c) Single-molecule conductance histogram for $\mathbf{1}$ and 2 measured in mesitylene $(1 \mathrm{mM})$ at $200 \mathrm{mV}$ bias (>5000 individual scans for each compound, with no data selection, compiled with 100 bins per decade, and normalised to the number of scans as counts/trace); (d) Transmission curves $\left(\log (T(E)) v s E-E_{F}^{D F T}\right)$ for $\mathbf{1}$ and $\mathbf{2}$ referenced to the Fermi level of the electrodes. In the case of the 6-oxoverdazyl radical $\mathbf{1}$, the transmission function $T(E)$ is the average transmission for both spin-up and spin-down contributions.

Single-molecule junctions of $\mathbf{1}$ and $\mathbf{2}$ were fabricated using the scanning tunnelling microscope break junction (STM-BJ) method in order to characterise their charge-transport properties. ${ }^{30}$ In this 
technique, an Au STM tip is driven into a Au substrate under DC bias $V$ until a metallic contact having conductance $\gg G_{0}$ is formed. The tip is then withdrawn at a constant speed of a few $\mathrm{nm} / \mathrm{s}$ in a dilute solution of the target molecular wire, while continuously monitoring the current $I$ at high acquisition rate $(>10 \mathrm{kS} / \mathrm{s})$ as a function of the relative tip-substrate position $z$. During the withdrawal process, the metallic contact is continuously thinned until it is reduced to a point contact having conductance $G_{0}$. As the withdrawal continues, the point contact will break, generating a nanogap of $\sim 0.5-0.6 \mathrm{~nm}$ due to snapback of the electrodes. ${ }^{31}$ Molecules from solution that have self-assembled along the metallic filament can bridge the resulting nanogap, thus fabricating the single-molecule junction. ${ }^{32}$ The withdrawal process is continued to stretch the molecule into its extended state and to finally rupture the molecular junction. The tip is then driven again into the substrate and the process is repeated several thousand times to acquire statistically significant data. Data are acquired in the form of $G(z)$ traces (conductance $G$ is calculated using Ohm's law $G=I / V$ ) that bear multiple plateaux at: (i) integer multiples of $G_{0}$, characteristic of conductance quantisation of single-channel conductors such as $\mathrm{Au}$ atoms; and (ii) at values $\ll G_{0}$, characteristics of transport through partially open quantum channels such as molecular wires. Data are then compiled in semilogarithmic histograms and heatmaps for further statistical analysis. Plateau in the $G(z)$ traces result in peaks in the histograms and in high-density areas in the heatmaps, yielding information on the most probable conductance values and molecular extension of the junction. Information about the instruments and materials ${ }^{33}$ used in this study and the data acquisition and analysis process ${ }^{34}$ can be found in the SI.

The 6-oxoverdazyl radical $\mathbf{1}$ displayed efficient charge transport, with a conductance peak centred at $10^{-3.4} G_{0}$, almost one order of magnitude more conductive than its closed-shell precursor $2\left(10^{-4.3} G_{0}\right)$ (experiments in mesitylene in Figure 2c; experiments in air available in the $\mathrm{SI}$ ). Further analysis shows that in all cases the molecule is stretched to its extended state in the junction, as features are evident in the heatmaps (available in the SI) up to an electrode separation of $\sim 0.65 \mathrm{~nm}(\sim 1.25 \mathrm{~nm}$ accounting for the snapback ${ }^{35}$ of the electrodes) that is in good agreement with the molecular length calculated using density-functional theory (DFT, Spartan '18, B3LYP/6-31G, $1.35 \mathrm{~nm}$ for 1 and 1.29 $\mathrm{nm}$ for 2 ).

To better understand these results, quantum transport calculations were used to compute the zerobias transmission probability for electrons with energy $E$ through junctions formed from $\mathbf{1}$ and $\mathbf{2}$. See 
the SI for detailed methods. A pair of sharp resonances in the transmission function $T(E)$, lying close to the Fermi level, are calculated for the junction formed from 1. These resonances arise from the singly-occupied (SOMO, $\alpha-\mathrm{HOMO}$, spin-up) and singly-unoccupied (SUMO, $\beta$-LUMO, spin-down) orbitals of the radical. The overlap of these resonances raises the value of $T(E)$ for 1 above $10^{-3}$ over a wide range of electron energies around the Fermi level. In contrast, the transport profile of the closed-shell tetrazin-3-one 2 shows that the Fermi level lies towards the bottom of a dip in the transmission function, within a wide HOMO-LUMO gap (Figure 2d).
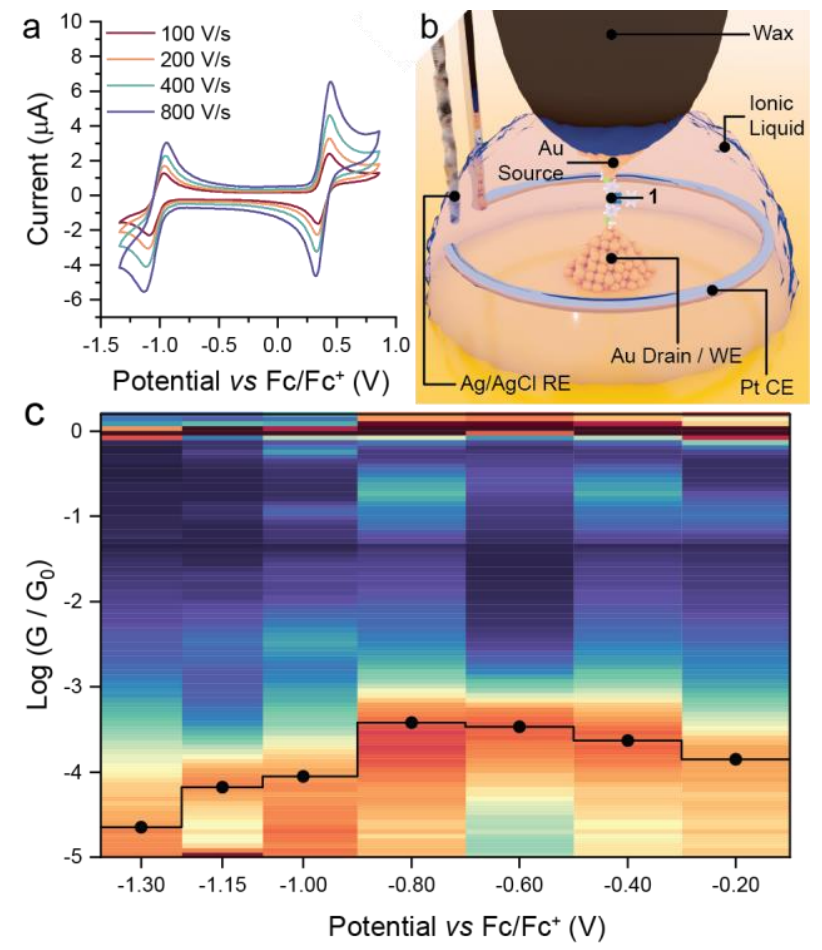

Figure 3: Electrochemical Experiments. (a) Cyclic voltammetry of 1 in degassed $\mathrm{CH}_{2} \mathrm{Cl}_{2}$, with $0.1 \mathrm{M}$ tetrabutylammonium hexafluorophosphate as supporting electrolyte. Potentials referenced to the ferrocene/ferrocenium redox couple using a decamethylferrocene standard. (b) Depiction of the 4electrode setup used in the single-molecule electrochemical gating studies with the molecular junction in place. (c) Heatmap of single-molecule conductance data across the electrochemical window explored, with gaussian fit of the peak position overlaid in black. Potential referenced to the ferrocene/ferrocenium redox couple as internal standard. Heatmap in (c) compiled with 10 bins per decade. More than 5500 individual scans were collected at each potential. Legend for (c): blue = low counts; red $=$ high counts.

As with other examples of 6-oxo-verdazyl radicals, ${ }^{28}$ compound 1 undergoes electrochemically reversible one-electron oxidation (to the corresponding monocation) and one-electron reduction (to the monoanion) processes (Figure 3a). To verify the involvement of the well-aligned singly-occupied orbital in the increased charge transport efficiency of $\mathbf{1}$, the electrical characteristics of the STM-BJ were further explored in an electrochemical environment, with a bipotentiostat maintaining a potential 
between the working electrode (the STM Au substrate and tip) and a Pt counter electrode vs an $\mathrm{Ag} / \mathrm{AgCl}$ reference electrode. The STM tip was insulated with Apiezon wax ${ }^{36}$ to reduce the faradaic current and constantly biased against the substrate by the bipotentiostat to ensure the two $\mathrm{Au}$ electrodes could act as source and drain (Figure 3b). Experimental measurements were made in the ionic liquid 1-butyl-3-methylimidazolium triflate, ${ }^{19,34,37}$ and under these conditions the setup displayed an open circuit potential of approximately $-0.4 \mathrm{~V} v s$ the ferrocene/ferrocenium $\left(\mathrm{Fc} / \mathrm{Fc}^{+}\right)$redox couple. The conductance of 1 remained at values around those obtained without electrochemical control from -0.2 to $-0.8 v s \mathrm{Fc} / \mathrm{Fc}^{+}$. In this potential window, a small electrostatic gating effect can be observed, with conductance slowly increasing as the potential is made more negative (Figure 3c). As the potential is further decreased to $-1 \mathrm{~V} v s \mathrm{Fc} / \mathrm{Fc}^{+}$, near to the reduction potential of $\mathbf{1}$, the junction conductance abruptly drops by almost one order of magnitude and continues to decrease as the potential is further reduced. Importantly, the more conductive open-shell state could be recovered by reversing the potential, highlighting the reversible transistor behaviour of junctions fabricated with $\mathbf{1}$ (see SI).

The initially observed electrostatic gating effect provides confirmation that $\mathbf{1}$ offers a resonance lying close to the Fermi level in the junction, with the abrupt decrease in junction conductance at more negative gate potentials indicating that this resonance is removed when $\mathbf{1}$ is reduced. In the potential window where 1 is in its open-shell state $\left(-0.2 \rightarrow-0.8 \vee v s \mathrm{Fc} / \mathrm{Fc}^{+}\right)$, conductance increases as the potential is made more negative, indicating that the transport resonance due to the SUMO (or $\beta$ LUMO) is initially at energy higher than the Fermi level of the electrodes. As the gate potential passes $-1.0 \mathrm{~V}$ vs $\mathrm{Fc} / \mathrm{Fc}^{+}$and $\mathbf{1}$ is reduced, the slope of the conductance/potential dependence suddenly changes sign, and the transport resonance is now due to the HOMO of the reduced $\mathbf{1}$, at energy significantly lower than the Fermi level. Attempts to obtain single-molecule conductance data at potentials $>0.5 \mathrm{~V} v s \mathrm{Fc} / \mathrm{Fc}^{+}$(where 1 would be oxidised to the monocationic state) were not possible due to increased instrumental noise at these potentials. We hypothesise this is due to the insolubility of the cationic species in the ionic liquid used in this study, which therefore deposits at the working electrode preventing further junction fabrication. Conductance data for $\mathbf{1}$ in the full electrochemical window explored $\left(-1.3 \rightarrow 0.5 \mathrm{~V}\right.$ vs $\left.\mathrm{Fc} / \mathrm{Fc}^{+}\right)$is provided in the $\mathrm{SI}$.

Having established that charge transport through $\mathbf{1}$ is enhanced by the presence of a partially filled molecular orbital near the Fermi level, attention was turned to single-molecule bias modulation 
experiments, with the aim of establishing the current-voltage $(I-V)$ behaviour of this compound. In these experiments, a modified STM-BJ technique was used. The tip was driven into the substrate and then retracted in a staircase manner, with steps of size commensurate to the length of the molecule of interest (in this case, $1.1 \mathrm{~nm}$ ). Between steps, the tip-substrate bias was held at a stable value (200 $\mathrm{mV}$ ) for $25 \mathrm{~ms}$, ramped to obtain the $\mathrm{I}-\mathrm{V}$ characteristics for $50 \mathrm{~ms}$, and again held stable for further 25 ms. Data were then sliced and processed using automated algorithms described in the Supporting Information. The $I-V$ characteristics of 1 are not linear, with the ohmic behaviour displayed between $\pm 0.7 \mathrm{~V}$ giving way to clear evidence of current rectification above $\pm 1.5 \mathrm{~V}$ (Figure $4 \mathrm{a}, \mathrm{b}$ ). At negative bias the current saturated at a value of $\sim 25 \mathrm{nA}$, while increasing rapidly to values $>100 \mathrm{nA}$ at positive bias, giving a rectification ratio $R R_{ \pm 2 V} \cong 5$. The tetrazine 2 showed no rectification or current saturation behaviour (see SI).
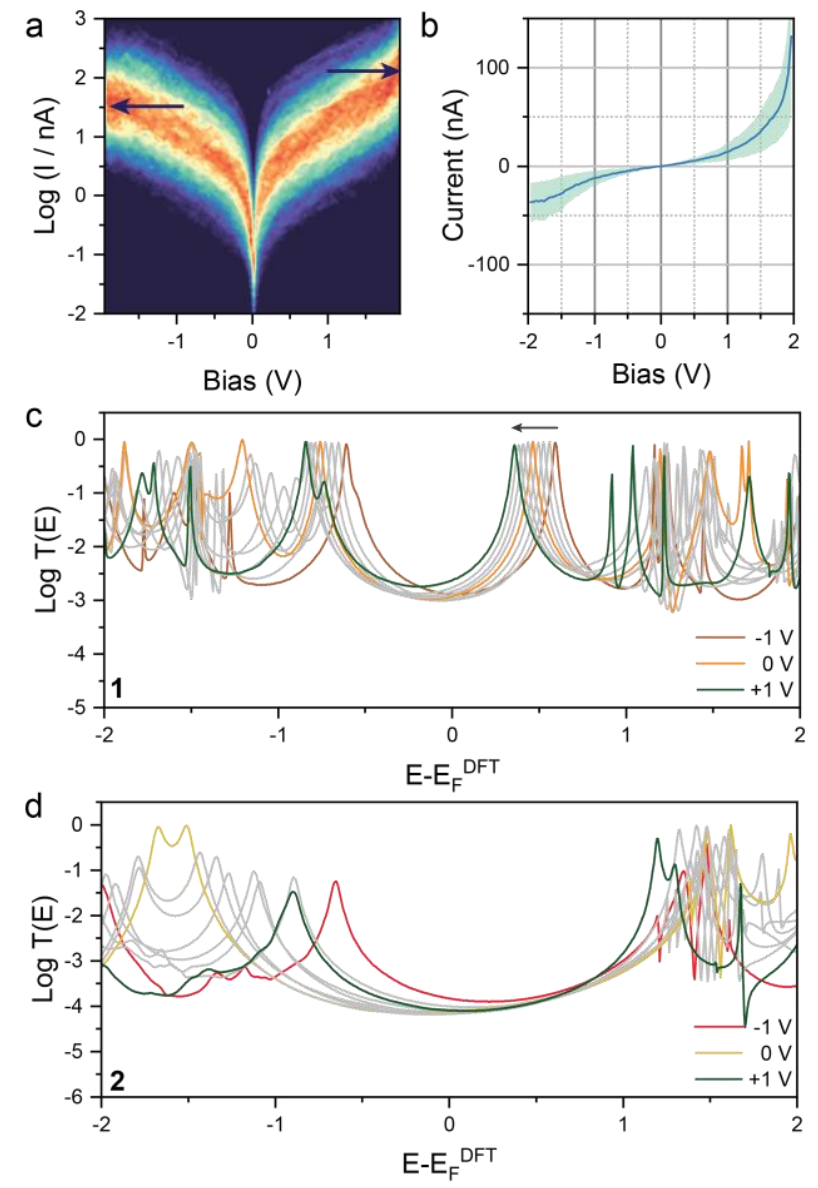

Figure 4: $I-V$ behaviour of 1 and 2. a) Semilogarithmic single-molecule $I-V$ heatmap for 1 . b) Gaussian fit of the data in (a), shown on a linear scale. Confidence interval is $\pm \sigma$ of the Gaussian fit. c) Bias-dependent transmission coefficient calculations for 1. (d) Bias-dependent transmission coefficient calculations for 2 . Data in (a) and (b) are compiled from $>10000$ traces, using the algorithms described in the SI. The heatmap in (a) has been compiled with 20 bins per decade and 50 bins per Volt. 
To better understand this phenomenon, we performed bias-dependent non-equilibrium Green's function calculations using the Gollum ${ }^{38}$ code, combined with the mean-field Hamiltonian obtained from DFT. These bias-dependent calculations demonstrate that the transmission spectrum is sensitive to the applied bias voltage, with the resonances shifting to lower energies when a positive voltage is applied, and conversely shifting to higher energies when negative bias is applied (see SI for more details). The transport resonance associated with the SUMO (or $\beta$-LUMO) of 1 enters the bias window at positive bias voltages (leading to high current) whereas it remains outside at negative bias voltages, due to its shift to higher energies (Figure 4c). These phenomena lead to a distinct asymmetry of the $I-V$ behaviour for the open-shell 1 . This rectifying behaviour demonstrated by a structurally symmetrical molecule therefore arises within the framework of a coherent tunnelling mechanism, and is not due to the surrounding environment, ${ }^{39,40}$ or different molecular interfaces to the source and drain electrodes, ${ }^{41-44}$ as in previously reported single-molecule diodes. For the closedshell 2, where the Fermi energy is close to the middle of the HOMO-LUMO gap, the shift of transmission spectra by bias voltage does not lead to significant difference of the transmission coefficient near $E_{F}$ at biases of opposite polarity, and therefore there is no asymmetry in the $I-V$ behaviour (Figure 4d).

In conclusion, these studies have demonstrated that the 6-oxoverdazyl radical 1 maintains its openshell character when incorporated into molecular junctions fabricated at room temperature. The openshell electronic character of $\mathbf{1}$ gives rise to a pair of additional transport resonances, arising from semi-occupied (SOMO or $\alpha$-HOMO) and semi-unoccupied (SUMO or $\beta$-LUMO) molecular orbitals. The energy alignment of these resonances with the Fermi levels of the metal electrodes grants 1 significantly enhanced charge-transport efficiency compared to its precursor 2 . The radical compound 1 can be reversibly, electrochemically reduced to a closed-shell anion within the junction, with the distinct charge-transport properties of the two charge states giving rise to single-molecule transistor behaviour. In addition, the bias-dependent behaviour of the SUMO transport resonance imparts rectifying behaviour to junctions fabricated with $\mathbf{1}$, with significantly greater current flowing under positive bias due to the migration of the transport resonances within the bias window. These results provide a strategy to incorporate radical compounds in single-molecule devices, key to enabling technologies such as molecular spintronics and molecular thermoelectrics. 


\section{Supplementary Information}

The supplementary information document contains details about the methods employed and additional single-molecule conductance data and calculations.

\section{Acknowledgements}

This work was supported by The Royal Society (RGSIR2l202119), EPSRC (EP/037765/1, EP/M005046/1, EP/M029522/1) and the Australian Research Council (DP190100073). A.V. also thanks the Royal Society for a University Research Fellowship (URF\R1\191241). H.S. acknowledges the UKRI for a Future Leaders Fellowship (MR/S015329/2). SS acknowledges the Leverhulme Trust for an Early Career Fellowship (ECF-2018-375). VJK gratefully acknowledges financial support from the Schlumberger Faculty for the Future scholarship program, and a Scholarship for International Research Fees from the University of Western Australia. RJN acknowledges funding from the Leverhulme Foundation (RPG-2019-308). EG gratefully acknowledges scholarship support from the Australian Research Council through DP190100074.

\section{Author Contributions}

PJL, SS and HS conceived the project. VJK and JZW synthesised the compounds used in this study and performed their characterisation. AV and RJN designed the charge transport experiments. SN performed the single-molecule experiments and analysed the data with software written by AV. SS performed the computational studies. EG performed preliminary single-molecule measurements. XQ trained SN in the electrochemical single-molecule experiments and assisted data acquisition. MJ and NC performed cw-EPR measurements. All authors contributed to the discussion of the results. AV, PJL and HS wrote the paper with contributions from all authors.

\section{Data Availability}

Raw single-molecule charge transport data, Labview Vis, and Python code used for its processing are available under a CC-BY license in the University of Liverpool Data Catalogue at:

- DOI: 10.17638/datacat.liverpool.ac.uk/1529 (STMBJ and EC-STMBJ measurements)

- DOI: $10.17638 /$ datacat.liverpool.ac.uk/1513 ( $I-V$ measurements). 


\section{Competing Financial Interests}

The authors declare no competing financial interests.

\section{References}

1. Gomberg, M. An Instance of Trivalent Carbon: Triphenylmethyl. J. Am. Chem. Soc. 22, 757771 (1900).

2. Ji, L., Shi, J., Wei, J., Yu, T. \& Huang, W. Air-Stable Organic Radicals: New-Generation Materials for Flexible Electronics? Adv. Mater. 32, 1-15 (2020).

3. Gryn'ova, G., Coote, M. L. \& Corminboeuf, C. Theory and practice of uncommon molecular electronic configurations. Wiley Interdiscip. Rev. Comput. Mol. Sci. 5, 440-459 (2015).

4. Lambert, C. J. Basic concepts of quantum interference and electron transport in singlemolecule electronics. Chem. Soc. Rev. 44, 875-888 (2015).

5. Wang, K. et al. Charge transfer complexation boosts molecular conductance through Fermi level pinning. Chem. Sci. 10, 2396-2403 (2019).

6. Sangtarash, S. \& Sadeghi, H. Radical enhancement of molecular thermoelectric efficiency. Nanoscale Adv. 2, 1031-1035 (2020).

7. Perrin, M. L. et al. Single-molecule functionality in electronic components based on orbital resonances. Phys. Chem. Chem. Phys. 22, 12849-12866 (2020).

8. Cui, L., Miao, R., Jiang, C., Meyhofer, E. \& Reddy, P. Perspective: Thermal and thermoelectric transport in molecular junctions. J. Chem. Phys. 146, 092201 (2017).

9. Miao, R. et al. Influence of Quantum Interference on the Thermoelectric Properties of Molecular Junctions. Nano Lett. 18, 5666-5672 (2018).

10. Mas-Torrent, M. et al. Organic radicals on surfaces: Towards molecular spintronics. J. Mater. Chem. 19, 1691-1695 (2009).

11. Herrmann, C., Solomon, G. C. \& Ratner, M. A. Organic Radicals As Spin Filters. J. Am. Chem. Soc. 132, 3682-3684 (2010). 
12. Low, J. Z. et al. The Environment-Dependent Behavior of the Blatter Radical at the MetalMolecule Interface. Nano Lett. 19, 2543-2548 (2019).

13. Bejarano, F. et al. Robust Organic Radical Molecular Junctions Using Acetylene Terminated Groups for C-Au Bond Formation. J. Am. Chem. Soc. 140, 1691-1696 (2018).

14. Frisenda, R. et al. Kondo Effect in a Neutral and Stable All Organic Radical Single Molecule Break Junction. Nano Lett. 15, 3109-3114 (2015).

15. Hayakawa, R. et al. Large Magnetoresistance in Single-Radical Molecular Junctions. Nano Lett. 16, 4960-4967 (2016).

16. Mugnaini, V. et al. Looking inside the perchlorinated trityl radical/metal spinterface through spectroscopy. J. Phys. Chem. Lett. 6, 2101-2106 (2015).

17. Paniagua, J. C. et al. Polychlorinated trityl radicals for dynamic nuclear polarization: the role of chlorine nuclei. Phys. Chem. Chem. Phys. 12, 5824 (2010).

18. Liu, J. et al. Radical-Enhanced Charge Transport in Single-Molecule Phenothiazine Electrical Junctions. Angew. Chem. Int. Ed. 56, 13061-13065 (2017).

19. Osorio, H. M. et al. Electrochemical Single-Molecule Transistors with Optimized Gate Coupling. J. Am. Chem. Soc. 137, 14319-14328 (2015).

20. Supur, M., Saxena, S. K. \& McCreery, R. L. Ion-Assisted Resonant Injection and Charge Storage in Carbon-Based Molecular Junctions. J. Am. Chem. Soc. 142, 11658-11662 (2020).

21. Li, J. et al. Reversible Switching of Molecular Conductance in Viologens is Controlled by the Electrochemical Environment. J. Phys. Chem. C 125, 21862-21872 (2021).

22. Haiss, W. et al. Redox State Dependence of Single Molecule Conductivity. J. Am. Chem. Soc. 125, 15294-15295 (2003).

23. Barr, C. L., Chase, P. A., Hicks, R. G., Lemaire, M. T. \& Stevens, C. L. Synthesis and Characterization of Verdazyl Radicals Bearing Pyridine or Pyrimidine Substituents: A New Family of Chelating Spin-Bearing Ligands. J. Org. Chem. 64, 8893-8897 (1999).

24. Kumar, V. J. et al. The syntheses, structures and spectroelectrochemical properties of 6-oxo- 
verdazyl derivatives bearing surface anchoring groups. Chemrxiv (2021) doi:10.26434/chemrxiv-2021-tqjwg.

25. Petunin, P. V. et al. Verdazyl Radical Building Blocks: Synthesis, Structure, and Sonogashira Cross-Coupling Reactions. European J. Org. Chem. 2018, 4802-4811 (2018).

26. Chemistnick, V., Chambers, D. \& Brook, D. J. R. Structure-property relationships of stable free radicals: Verdazyls with electron-rich aryl substituents. J. Org. Chem. 74, 1850-1857 (2009).

27. Neugebauer, F. A., Fischer, H. \& Krieger, C. Verdazyls. Part 33. EPR and ENDOR studies of 6-oxo- and 6-thioxoverdazyls. X-Ray molecular structure of 1,3,5-triphenyl-6-oxoverdazyl and 3-tert-butyl-1,5-diphenyl-6-thioxoverdazyl. J. Chem. Soc. Perkin Trans. 2535 (1993) doi:10.1039/p29930000535.

28. Gilroy, J. B., McKinnon, S. D. J., Koivisto, B. D. \& Hicks, R. G. Electrochemical studies of verdazyl radicals. Org. Lett. 9, 4837-4840 (2007).

29. Fuller, R. O. et al. Enhanced synthesis of oxo-verdazyl radicals bearing sterically-and electronically-diverse C3-substituents. Org. Biomol. Chem. 19, 10120-10138 (2021).

30. Xu, B. \& Tao, N. Measurement of Single-Molecule Resistance by Repeated Formation of Molecular Junctions. Science 301, 1221-1223 (2003).

31. Hong, W. et al. Single molecular conductance of tolanes: experimental and theoretical study on the junction evolution dependent on the anchoring group. J. Am. Chem. Soc. 134, $2292-$ 304 (2012).

32. Fu, T., Frommer, K., Nuckolls, C. \& Venkataraman, L. Single-Molecule Junction Formation in Break-Junction Measurements. J. Phys. Chem. Lett. 12, 10802-10807 (2021).

33. Wu, C. et al. Folding a Single-Molecule Junction. Nano Lett. 20, 7980-7986 (2020).

34. Wu, C. et al. A Chemically Soldered Polyoxometalate Single-Molecule Transistor. Angew. Chem. Int. Ed. 59, 12029-12034 (2020).

35. Quek, S. Y. et al. Mechanically controlled binary conductance switching of a single-molecule junction. Nat. Nanotechnol. 4, 230-234 (2009). 
36. Kazinczi, R., Szõcs, E., Kálmán, E. \& Nagy, P. Novel methods for preparing EC STM tips. Appl. Phys. A Mater. Sci. Process. 66, S535-S538 (1998).

37. Kay, N. J. et al. Single-molecule electrochemical gating in ionic liquids. J. Am. Chem. Soc. 134, 16817-16826 (2012).

38. Ferrer, J. et al. GOLLUM: a next-generation simulation tool for electron, thermal and spin transport. New J. Phys. 16, 093029 (2014).

39. Capozzi, B. et al. Single-molecule diodes with high rectification ratios through environmental control. Nat. Nanotechnol. 10, 522-527 (2015).

40. Atesci, H. et al. Humidity-controlled rectification switching in ruthenium-complex molecular junctions. Nat. Nanotechnol. 13, 117-121 (2018).

41. Gao, D. et al. Fabrication of asymmetric molecular junctions by the oriented assembly of dithiocarbamate rectifiers. J. Am. Chem. Soc. 133, 5921-30 (2011).

42. Díez-Pérez, I. et al. Rectification and stability of a single molecular diode with controlled orientation. Nat. Chem. 1, 635-641 (2009).

43. Vezzoli, A. et al. Single-Molecule Transport at a Rectifying GaAs Contact. Nano Lett. 17, 1109-1115 (2017).

44. Aragonès, A. C. et al. Single-molecule electrical contacts on silicon electrodes under ambient conditions. Nat. Commun. 8, 15056 (2017). 